\title{
DIAGNOSTIC AND PROGNOSTIC SIGNIFICANCE OF BONE MARROW BIOPSY IN NON HODGKINS LYMPHOMA
}

\author{
${ }^{1}$ Ahmad Mokhtar Ahmad, ${ }^{1}$ Saad AL Deen Sabery Esh, ${ }^{2}$ Amira Mokhtar Khorshed, ${ }^{1}$ Mohamad \\ Hossam AL Deen Mourad \\ ${ }^{1}$ Clinical Pathology Department, Zagazig University, Zagazig, Egypt \\ ${ }^{2}$ Clinical Pathology Department, NCI, Cairo University, Egypt
}

Corresponding Auther: Ahmad Mokhtar Ahmad Ibrahem \& Tel.: $01222222430 \&$

Email: abo_cpyahoo.com

\begin{abstract}
Background: Trephine bone marrow biopsy is the standard method for the diagnosis of neoplasia. In the last few years its role has been defined further by the widespread introduction of immunohistochemistry (IHC), the method that increased the diagnostic power of the trephine bone marrow biopsy by detecting specific antigens in the tumor tissue using nonflourescent chromogens that can be seen by conventional microscopy. Objective: to evaluate the diagnostic and prognostic significance of bone marrow biopsy in non-Hodgkin's lymphomas. Correlation of bone marrow biopsy finding with other prognostic markers in non-Hodgkin's lymphomas is another aim. Methods: The study was performed on 50 patients of NHL diagnosed by LN histopathology. All patients were subjected to: clinical examination and laboratory investigations including complete blood picture, liver function tests, kidney function tests, Erythrocyte sedimentation rate, serum lactate dehydrogenase, B2 microglobulin, bone marrow aspiration, immunophenotyping of bone marrow aspiration of the disseminated cases, bone marrow trephine biopsy for: histological classification according to WHO, staging of NHL and Immunohistochemical staining for CD3 and CD20. Results: Twenty patients $(40 \%)$ positive for infiltration on examination of marrow biopsy and 30 patients $(60 \%)$ were negative. The results of fifty NHL cases showed only in six cases, both bone marrow biopsy and aspiration were positive $(12 \% ; 95 \% \mathrm{CI}, 4.0: 22.0)$, and in 30 out of 50 , the two parameters were negative $(60 \%, 95 \% \mathrm{CI}, 46: 54)$. Thus, the agreement level between BMB and BMA in the diagnosis of NHL was $V Y \%$ and discrepancies between bone marrow biopsy and aspirate were found in the remainder. In 14 of 50 specimens, BMB alone was positive (28\%; 95\% CI, 16 : 42). Conclusion: Bone marrow biopsy morphology and immunohistochemical staining remain the cornerstone for proper staging in NHL patients to increase the diagnostic and prognostic accuracy, for appropriate treatment protocols.

Key words: NHL, non Hodgkin's lymphoma, BMB, Bone marrow biopsy. BMA, bone marrow aspiration and IHC, immunohistochemistry, diagnosis, prognosis.
\end{abstract}

\section{INTRODUCTION}

$I^{\prime}$ n Egypt, the fifth most common malignancy in both the sexes is nonHodgkin's lymphoma. The general incidence rate in (1995) was $5.9 \%$ and reached $8.99 \%$ in (2004), with a peak in (2002) where it was $9.4 \%$. The male incidence showed a clear rise from the year (1995) to (1998), with a slowing from 1999 to 2002, followed by another peak from (2003). Women showed an obvious increased incidence rates from the year
1995 to 2000 , then a decrease in incidence afterwards till (2004) [ 1].

Non-Hodgkin's lymphoma accounts for about $90 \%$ of all lymphomas where the remaining ten percent are known as Hodgkin lymphoma. Non-Hodgkin lymphomas have a wide range of histopathological appearances and clinical features at presentation, which can make the diagnosis very difficult. Early diagnosis of lymphoma is essential and critical due to the effective, and mostly 
curative therapies are available for many subtypes of lymphoma when early early diagnosed, also at early diagnosis only local treatment may be the only needed for treatment [2].

This group of cancers developed primarly in the lymph nodes, but can occur in almost any other tissue as thyroid glands, bone or bone marrow, and ranges from the more indolent follicular lymphoma, to the more aggressive lymphomas as Burkitt's lymphomas and diffuse large B-cell lymphoma. About $85-90 \%$ originate from B lymphocytes; the remainders arise from T- lymphocytes or NK lymphocytes. classification systems grouped these malignancies according to their histological characteristics. The most reliable system is the fourth edition of the WHO classification of tumours of haemopoietic and lymphoid tissues, in (2008) [3].

Many patterns of infiltration of bone marrow in non-Hodgkin lymphomas are documented and may show more than one pattern of bone marrow infiltration in an individual patient. These patterns are not only helpful to identify malignant lymphoid infiltrates but also aids in classification of the lymphoma. The patterns of infiltrates in the bone marrow occur in any one or a combination of five different patterns: focal random; focal paratrabecular; interstitial; diffuse; and intrasinusoidal.[4]

The aspirate and trephine biopsy specimens are usually complementary to each other in many hematological and non hematological disorders, but bone marrow biopsy is critical and more diagnostic in hematological disorders with focal infiltration as in lymphomas and in bone marrow metastasis. So when both are obtained, they provide a complete evaluation of the bone marrow. The interpretation of results requires the integration of peripheral blood, $\mathrm{CBC}$, bone marrow aspirate and trephine biopsy findings, together with the results of supplementary tests such as immunohistochemistry of bone marrow biopsy, immunophenotyping of aspirate sample, cytogenetic analysis and molecular genetic studies, in the context of clinical and other diagnostic and prognostic findings. [5].

\section{Subjects and Methods}

The study was carried out in the period between January 2013 to January 2015 after approval of the Institutional Review Board (IRB) . A written informed consent was obtained from all subjects after complete description of the study. All cases were recruited from the bone marrow biopsy unit (Hematology section), Clinical pathology department, National cancer Institutes, Cairo university.

The present study included 50 patients of non Hodgkin's lymphoma diagnosed by LN biopsy.

\section{Methods:}

I- Patients were routinely subjected to the following:

Complete Clinical examination, Complete blood picture, Liver function tests, kidney function tests, serum lactate dehydrogenase enzyme, LDH and B2 microglobulin, B2M, Bone marrow aspiration and immunophenotyping of BMA samples, in disseminated cases. Bone marrow trephine biopsy for the following tests: Histology classification will be based on the 1999 world Health Organization classification of lymphoma. Staging will be performed according to the Ann- Arbor criteria and CD3 and CD20 by Immunohistochemical staining of BMB specimens (DAKO diagnostic).

\section{II- Technique of bone marrow Biopsy sampling:}

The BM biopsy was obtained from the posterior superior iliac spine (PSIS) using the trephine biopsy needles (Jamshidi needles).

The patient was placed in the left lateral position with the knee drawn up to the chest. The PSIS were located by thumbnail pressure then the skin was sterilized with topical antiseptic. The skin, subcutaneous tissue and periosteum of the marked area were infiltrated with local anesthetic (xylocain 2\%). After a brief delay (3-5 min), using Islam needle, bone marrow 
aspiration sample first obtained. The sample of aspirate put on EDTA tube for immunophenotyping and adequate numbers of slides directly from the needle of aspirate is prepared, dried to be stained by Lishman stain.

Then, with a scalpel blade a small 3-4 mm incision was made at the same site of aspiration. The needle was held with the proximal end in the palm and the index finger against the shaft near its tip. When the stylet locked in place the needle was introduced through the incision pointing toward the anterior superior iliac Spine and was brought into contact with PSIS. Using gentle but firm pressure, the needle was advanced and rotated in clockwise counter clockwise motion. Penetration into the marrow cavity was sensed by decreased resistance to the forward movement of the needle. The stylet was then removed and the needle was advanced slowly and gently, with a twisting motion around its long axis, to a depth of 3 to 4 $\mathrm{cm}$ until adequate marrow core was obtained. The stylet is used to show the length of the core and when appropriate length is reached the needle was rotated several times on its axis and pulled back about $3 \mathrm{~mm}$, and then with less pressure, the needle was reinserted to the original depth but directed at slightly different angle to cut the core. Again the needle was rotated several times along its axis with full twist to the right and left in order to free the core from is attachment in the marrow cavity. The needle was then withdrawn and the core inside it was removed by inserting the probe through the cutting tip and extruding the specimen through the needle on a clean glass slide making touch imprint between tow glass slides. The core was then preserved in $10 \%$ formalin.

\section{Preparations done for the BM biopsy} core:

The obtained BMB core was immediately preserved in capped plastic tube, containing $10 \%$ Formol-Saline for 24-48 hours in room temperature. The fixed specimen was then washed in slowly running tap water for a minimum of 30 minutes. BMB core was placed in Formic Acid- Sodium Citrate for 2 days. When decalcification was completed, the core was transferred directly to $70 \%$ alcohol. The decalcified core (placed into tissue embedding cassette) was transported into a wire-mesh basket, where it was dehydrated in ascending grades of

( 70- 80- 90-100\%) alcohol, cleared in Xylene, impregnated and embedded in paraffin wax. On a rotating microtome, the

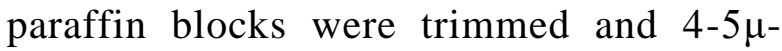
thick sections were obtained and mounted onto glass slides using a floating water bath $\left(40-45^{\circ} \mathrm{C}\right)$.

\section{Haematoxylin \& Eosin Staining:}

Sections were deparaffinized in 3 changes of Xylene, 10 minutes each then rehydrated in 2 changes of $100 \%$ ethyl alcohol, 5 minutes each then $90 \%$ alcohol, then $80 \%$ alcohol, then $70 \%$ ethyl alcohol for 2 minutes each and were rinsed briefly in Distilled Water (DW). Sections were stained with Harris haematoxylin solution for 2-5 minutes then were washed in running tap water for 2-3 minutes. Counterstain in Eosin $\mathrm{Y}$ working solution was used for 1 minute. Over stain was washed off in DW. Dehydration through $95 \%$ alcohol \& 2 changes of $100 \%$ alcohols, 5 minutes each was done. Then sections were cleared in 2 changes of Xylene, 5 minutes each.

IV- Immunohistochemical staining by DAKO Cytomation Envision[6].

To detect the type of infiltration and discordance with the primary site, panel of Monoclonal Antibodies (DAKO, USA) was performed including CD20 and CD3. Preparation done for any formalin fixed, paraffin embedded marrow section before any type of staining as above was done. After that: Ag Retrieval was done by: heat induced epitope retrieval (HIER):Buffer: Dako Target Retrieval sol. (Conc. 10x ) was diluted 1:10, so $1 \mathrm{ml}$ conc. Sol. to $10 \mathrm{ml}$ D.W. , cover the section, 45 min. Blocking endogenous peroxidase was done by applying enough blocking reagent to fully cover the sections for 10 minutes. The preparation was rinsed 
gently in 3 changes of PBS (PH6, $10 \mathrm{x}$, PH9, 10x and PH8, 100x); 10-20 dipping each, then excess buffer was tapped off and wiped carefully around. 1ry Antibodies were diluted by freshly prepared clean PBS, according to dilution ranges recommended in the MoAb data sheet, then each Ab Eppendorf was mixed well by Vortexing ( 3 times), then enough diluted $\mathrm{Ab}$ was applied on its corresponding labeled section then incubated in closed humid chamber for 45 minutes. The preparation was rinsed gently in 3 changes of PBS; 20 dipping each, then excess buffer was tapped off and wiped carefully around the sections. Enough "labeled Polymer" was applied, conjugated with 2 nd $\mathrm{Ab}$, to cover the whole sections, then incubated for 40 minutes. The preparation was rinsed gently in 3 changes of PBS; 20 dipping each, then sections were leaved in the 3rd change of PBS till preparation of DAB. Freshly prepared substrate chromogen solution by adding $20 \mu \mathrm{l}$ DAB-chromogen to $1000 \mu \mathrm{l}$ bufferedsubstrate was done, then mixing well by Vortex was done, and then left in Dark. Returning to sections in the 3rd change of PBS excess buffer was tapped off and wiped carefully around the sections, and then enough of prepared DAB chromogensubstrate solution was applied, and then incubated in closed Dark chamber, for 10 minutes. The preparation was rinsed gently in 3 changes of PBS; 20 dipping each, then excess buffer was tapped off and wiped carefully around the sections.Counter stain was performed by immersing sections, in (Hx) stain, for ten min. Washing in D.W was done. Dehydration by short fast (continuous
2 dipping) in ascending grades of ethyl alcohols (70 -80-90-100\%) was done, then clearing by 2 dipping in 2 changes of Xylene, then the preparation was left to dry.Mount with DPX was done, and then clean glass cover was used, then left to dry.Results: Positive results appear brown.

\section{Statistical analysis}

The results were collected, tabulated, statistically analyzed by IBM personal computer and statistical package SPSS version 19. Descriptive statistics: mean (x) and standard deviation (SD) and analytic statistics (e.g. Chi square, Mann whiteny test and confidence interval) to study the association between two qualitative and quantitative variables were used.

\section{Results}

This study conducted on fifty patients previously diagnosed as non Hodgkin's lymphoma by LN histopathology, 19 $(38.0 \%)$ were females and $31(62.0 \%)$ were males with Male to Female ratio:1.6:1\%. The median age of studied cases was 55.5 years with a range of 5 years and 77 years. Twenty patients $(40 \%)$ were positive for infiltration on examination of marrow biopsy and 30 patients $(60 \%)$ were negative. The results of bone marrow aspiration were, only six cases $(6 / 20,30 \%)$ of the positive bone marrow biopsy cases were positive on examination of bone marrow aspiration. The most common Cellularity pattern of bone marrow biopsy was the normocellular pattern $(12 / 20$, $60 \%)$ of the positive $\mathrm{BMB}$ results and $(25 / 30,83.3 \%)$ of the negative BMB results.

Table (1): Comparison of platelets count, hemoglobin level and TLC between positive and negative BMB cases:

\begin{tabular}{|c|c|c|c|c|}
\hline \multicolumn{2}{|c|}{$\overline{\text { BMB infiltration }}$} & $\begin{array}{c}\text { TLC } \\
(\mathbf{x ~ 1 0} / \mathbf{u l})\end{array}$ & $\begin{array}{c}\text { Platelets count } \\
\left(\times 10^{3} / \mathrm{ul}\right)\end{array}$ & $\begin{array}{c}\text { Hemoglobin } \\
\text { (g/dL) }\end{array}$ \\
\hline \multirow[t]{2}{*}{ Positive } & Mean \pm SD & $8.75 \pm 8.06$ & $181.5 \pm 88.1$ & $10.6 \pm 1.33$ \\
\hline & Median & V.YY & 171.5 & 9.75 \\
\hline \multirow[t]{2}{*}{ Negative } & Mean \pm SD & $7.09 \pm 4.2$ & $188.2 \pm 79.0$ & $11.47 \pm 1.44$ \\
\hline & Median & 5.7 & 176 & 11.1 \\
\hline$P$ value & & $0.51(\mathrm{NS})$ & $0.53(\mathrm{NS})$ & $<0.001(\mathrm{HS})$ \\
\hline
\end{tabular}


Table (2): Comparison of B- symptoms, splenomegaly, hepatomegaly and IPI between between positive and negative BMB cases

\begin{tabular}{|c|c|c|c|c|c|c|}
\hline & \multicolumn{4}{|c|}{ BMB } & & \multirow{3}{*}{ P-value } \\
\hline & \multicolumn{2}{|c|}{$\begin{array}{r}\text { Positive } \\
(\text { no=20) }\end{array}$} & \multicolumn{2}{|c|}{$\begin{array}{c}\text { Negative } \\
(\text { no=30) }\end{array}$} & & \\
\hline & No & $\%$ & No & $\%$ & $\mathbf{X} 2$ & \\
\hline \multicolumn{7}{|l|}{ B-Symptoms } \\
\hline Positive & 11.0 & $55 \%$ & 14.0 & $46.7 \%$ & 0.33 & 0.387 \\
\hline Negative & 9.0 & $45 \%$ & 16.0 & $53.3 \%$ & & NS \\
\hline \multicolumn{7}{|l|}{ Splenomegaly } \\
\hline Present & 15.0 & $75 \%$ & 16.0 & $53.3 \%$ & 2.39 & 0.105 \\
\hline Absent & 5.0 & $25 \%$ & 14.0 & $46.7 \%$ & & NS \\
\hline \multicolumn{7}{|l|}{ Hepatomegaly } \\
\hline Present & 8 & $40 \%$ & 13.0 & $43.3 \%$ & 0.05 & 0.52 \\
\hline Absent & 12.0 & $25 \%$ & 17.0 & $46.7 \%$ & & NS \\
\hline \multicolumn{7}{|l|}{ IPI } \\
\hline IPI $<2$ & 2.0 & $10 \%$ & 22.0 & $73.3 \%$ & 19.2 & $<0.001 * *$ \\
\hline IPI > 2 & 18.0 & $90 \%$ & $\overline{8.0}$ & 26.7 & & HS \\
\hline
\end{tabular}

There was statistically high significant difference between positive and negative bone marrow biopsy studied cases as regard Serum LDH and ESR, $(\mathrm{P}<0.001)$. There were statistically significant difference between positive and negative bone marrow biopsy studied cases as regard Serum B2M (P: <0.05).

Table (3): Bone marrow results as regard the bone marrow biopsy (BMB) among studied cases.

\begin{tabular}{lcc}
\hline $\begin{array}{l}\text { BMB infiltration by } \\
\text { NHL }\end{array}$ & Number & Percent \\
\hline Positive & 20 & $40 \%$ \\
\hline Negative & 30 & $60 \%$ \\
\hline
\end{tabular}

Table (4): Incidence of bone marrow infiltration in different NHL histological sub types.

\begin{tabular}{lll}
\hline Pathological subtypes: $\mathbf{n}(\%)$ & $\begin{array}{l}\text { Positive infiltration } \\
\text { No: } 20\end{array}$ & $\begin{array}{l}\text { Negative infiltration } \\
\text { No: } 30\end{array}$ \\
\hline DLBCL: $40(80 \%)$ & $11(55 \%)$ & $29(96.7 \%)$ \\
\hline MCL: 3.0 (6.0\%) & $3.0(15 \%)$ & 0.0 \\
\hline Burkitts lymphoma: $2.0(4.0 \%)$ & $1.0(5.0 \%)$ & $1.0(3.3 \%)$ \\
\hline T-NHL: $2.0(4.0 \%)$ & $2.0(10 \%)$ & 0.0 \\
\hline MZL: $1.0(2.0 \%)$ & $1.0(5.0 \%)$ & 0.0 \\
\hline Follicular Lymphoma: 1.0 (2.0\%) & $1.0(5.0 \%)$ & $\mathbf{0 . 0}$ \\
\hline Lymphoplasmacytic lymphoma1.0 & $1.0(5.0 \%)$ & $\mathbf{0 . 0}$
\end{tabular}
$(2 \%)$

B-NHL had the high incidence $(18 / 20 ., 90 \%)$ of the positive bone marrow biopsy results and only two cases $(2 / 20 ., 10 \%)$ were T-NHL. Patients with positive infiltration in bone marrow biopsy, CD20 positive (by IHC) were $(18 / 20,90 \%)$ and only tow cases with negative CD20 $(2 / 20,10 \%)$. Among the positive bone marrow biopsy cases, CD 3 positive (by IHC) were $(2 / 20,10 \%)$ and 18 cases $(18 / 20,90 \%)$ were negative CD3 (by IHC). 
Table (5): Patterns of BM infiltration among positive BMB studied cases

Pattern Number Percent

\begin{tabular}{lll}
\hline Interstitial & $\mathbf{1 0}$ & $\mathbf{5 0 \%}$ \\
\hline Diffuse & $\mathbf{5}$ & $\mathbf{2 5 \%}$ \\
\hline Nodular & 1 & $\mathbf{5 \%}$ \\
\hline Paratrabecular & 4 & $\mathbf{2 0 \%}$ \\
\hline
\end{tabular}

Table (6): Confidence interval (CI) of bone marrow biopsy compared to the bone marrow aspiration among studied cases:

\begin{tabular}{lllllc}
\hline \multicolumn{2}{c}{ Group } & Number & $\%$ & \multicolumn{2}{c}{$95 \%$ CI } \\
\hline 1 & +ve BMB, +ve BMA & $6 / 50$ & $12 \%$ & 4 & $: 22$ \\
\hline$r$ & +ve BMB, -ve BMA & $14 / 50$ & $28 \%$ & 16 & $: 42$ \\
\hline$r$ & -ve BMB, -ve BMA & $30 / 50$ & $60 \%$ & & $46: 74$
\end{tabular}

Only Six cases of the 50 (12\%) specimens of NHL the parameters were positive for infiltration by lymphoma, and in 30 of fifty $(60 \%)$ the parameters were negative. Thus, the agreement level between the methods in diagnosis of NHL was $72 \%$ and the discrepancies between them were found in the remainder. BMB alone was positive in fourteen patients $(28 \%)$.
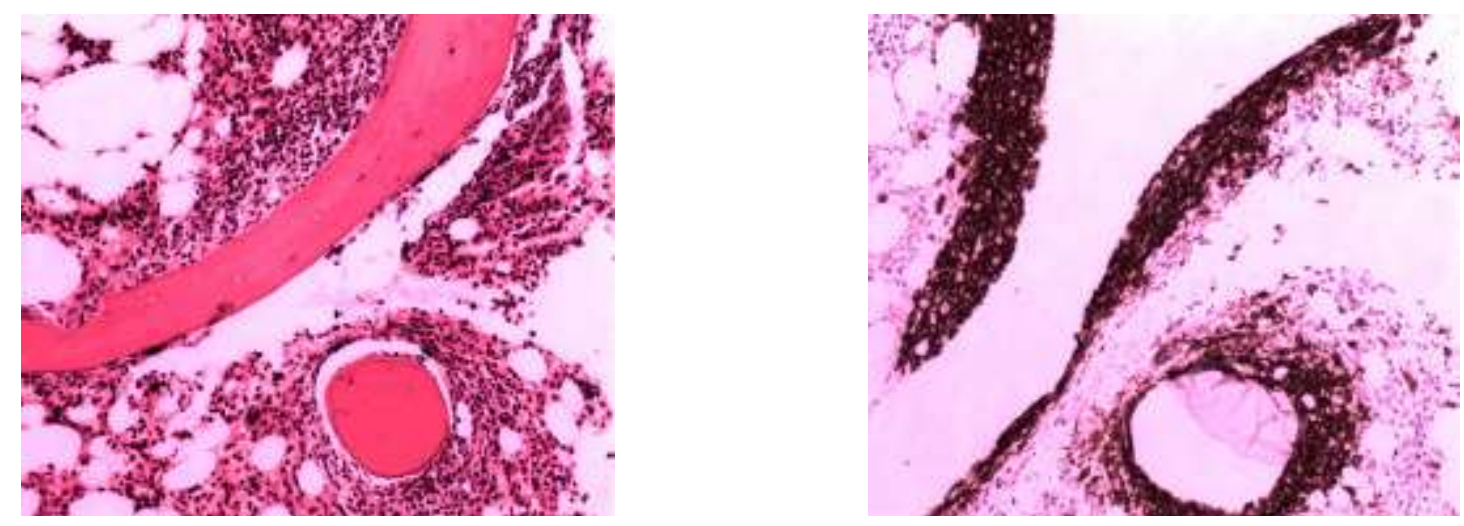

\section{(A): FL (H\&E)}

Fig (1): A case of FL:, (A):Bone marrow biopsy H\&E, (B): CD20 positive by IHC

\section{DISCUSSION}

In the current work, the majority of cases are B-cell non Hodgkin's lymphoma 48/50 (96\%) cases were B-NHL while only 2/50 (4\%) cases were T-NHL which was the same as observed by Sovani et al., and Kumar et al., where the incidence of B cell lymphomas was $90 \%, 85 \%$ and $\mathrm{T}$ cell lymphomas $10 \%, 15 \%$ respectively. Also in another work by EL Bolkeney et al., 2009 where the incidence of B-cell phenotype NHL was the major type ( 98\%) and the T-cell NHL phenotype was only $(2 \%)[7,8]$.
Also Elrefaey et al., reported that the incidence of the B cell phenotype of NHL was $(88 \%)$ and $\mathrm{T}$ cell phenotype of NHL was (12\%).[9]

In this study, the incidence of positive bone marrow infiltration proved by results of bone marrow biopsy, BMB, were $20 / 50(40 \%)$ and $30 / 50(60 \%)$ were negative. These results were similar a study by EL Bolkeney et al., where the incidence of bone marrow infiltration was $40.8 \%$.[8]

In other work by Kumar et al., reported that, $27 / 49$, $(55.1 \%)$ of NHL cases showed infiltration of marrow.[10] 
In other study by Elrefaey et al., bone marrow was infiltrated by lymphoma in $18 \%$ of patients this variation can be attributed to inclusion of unequal proportions of patients with early and advanced disease. In addition, the inclusion of different proportions of various histological subtypes of lymphomas in the different studies may account for this variation as the incidence of BM pathology varies greatly according to the histological subtypes. [9]

In the present study, the most common type of NHL was diffuse large B- cell lymphoma, DLBCL, where 40/50 (80\%) were DLBCL with only $11 / 40$ cases $(27.5 \%)$ were positive in bone marrow biopsy and 29/40 cases $(72.5 \%)$ of DLBCL were negative for infiltration of bone marrow. Followed by three cases 3/50 (6\%) diagnosed as MCL with all the three cases were positive for infiltration of bone marrow. 3/20 (15\%). Tow cases 2/50 (10\%) were Brukitts lymphoma one case showed positive infiltration of bone marrow $(1 / 20 ., 5.0 \%)$ and one case were negative for infiltration of bone marrow (1/30, 3.3\%). Tow cases were T-NHL (2/50., $10 \%)$ and the two cases showed positive infiltration of bone marrow (2/20., 10\%). One case was marginal zone lymphoma, MZL $(1 / 50 ., 2.0 \%)$ and was positive for infiltration of bone marrow $(1 / 20 ., 5.0 \%)$. One case was follicular lymphoma, (1/50., and $2.0 \%)$ and was positive for infiltration of bone marrow $(1 / 20 ., \quad 5.0 \%)$. One case was Lymphoplasmacytic lymphoma (1/50., 2.0\%) and was positive for infiltration of bone marrow $(1 / 20$., $5.0 \%)$. This agreement with other work by Chung et al., who reported that DLBL is the most common type to invade the bone marrow ( $27 \%$ of patients with DLBL).

In other study by EL Bolkeney et al., who report that the incidence of DLBCL that invade the bone marrow was $31.1 \%$ of all cases of DLBCL.[8]

Also in a study by Kumar et al., who report that the incidence of DLBCL in his work was $9 / 19(47.37 \%)$ that invade the bone marrow.[10]

In other work by Elrefaey et al., DLBCL cases that showed infiltration of bone marrow were $8 / 59(13.5 \%)$.[9]
Also study by Campbell et al., who reported that $(11 \%)$ of DLBCL involved the marrow. [11]

In other work by Sovani et al., reported the follicular lymphoma as the commonest lymphoma to invade the marrow $26.2 \%$ followed by Lymphoplasmacytic $19.2 \%$ and DLBL 12.5\%. [7]

Another study by Arber and George, reported that follicular lymphoma (37.5\%) was the most common type to involve the marrow, followed by diffuse large B-cell lymphoma $(16.0 \%)$. This difference can be attributed due to the high number of Follicular lymphoma included in Sovani and Arber studies and also unequal proportions of patients with early and advanced stage of non Hodgkin's lymphoma.[12]

In the present study the predominant histological pattern of involvement by lymphoma cells was interstitial, (10/20, 50\%), followed by diffuse pattern $(5 / 20,25.0 \%)$, paratrabecular $(4 / 20,20.0 \%)$ and the nodular pattern $(1 / 20,5.0 \%)$.this results were in agreement with that reported by Elrefaey et al., where the most predominant histological pattern of involvement by a lymphomatous infiltrate was interstitial $77.8 \%$, followed by diffuse pattern $11.1 \%$, paratrabecular $5.6 \%$ and nodular pattern 5.6\%.[9].

Also in other study by Sah et al., where commonest pattern of infiltration was interstitial $42 \%$ followed by diffuse pattern 20\%.[13].

But the results in the present study varied from the findings of Kumar et al, where the predominant histological pattern observed was mixed $52 \%$, followed by nodular $22 \%$, paratrabecular $11 \%$. Increased mixed pattern of (nodular and interstitial) in Kumar et al., study can be also attributed to the inclusion of high number of CLL patients.[10,13]

In the current study 15/20 (75\%) patients had splenomegaly showed bone marrow infiltration. $8 / 20(40 \%)$ of patients with hepatomegaly showed bone marrow infiltration. Difference between patients with or without organomegaly was statistically non-significant regarding bone marrow infiltration (p: 0.105 and p: 0.5 respectively). 
In other study by Elrefaey et al., Four/16 $(25 \%)$ patients had splenomegaly showed bone marrow infiltration. Four/15 (26.7\%) of patients with Hepatomegaly showed bone marrow infiltration. Difference between patients with or without organomegaly was statistically non-significant regarding $\mathrm{BM}$ infiltration ( $\mathrm{p}$ : 0.426 , p: 0.343 respectively). This difference in results may be due to unequal proportion of population with different stage of non Hodgkin's lymphoma[9] In the current study, patients with positive Bsymptoms showed bone marrow infiltration were $(55 \%)$ and patients with negative B symptoms were $(45 \%)$. Difference between patients with or without B- symptoms was statistically non-significant as regards bone marrow infiltration ( $\mathrm{p}: 0.387$ ).

These results were incorcordance to that of a study by EL Bolkeney et al., where $60 \%$ of patients with positive marrow infiltration was positive for B-symptoms. [8]

In a study by Elrefaey et al.., (17\%) patients with B- symptoms showed bone marrow infiltration versus $(83 \%)$ patients with no B symptoms showed no infiltration. Difference between patients with or without B symptoms was statistically non-significant regarding bone marrow infiltration (p: 0.9). [9].

In the present work, patients with marrow infiltration as regarded revised international prognostic index (IPI) were divided into these with IPI less than score two $(10 \%)$ and IPI > 2 (90\%). There was statistically high significant difference between positive and negative bone marrow biopsy studied cases as regard IPI, $(\mathrm{P}<$ $0.0001)$. That also reflects the importance of bone marrow biopsy results in the prognosis of lymphoma.

This data is in concordance with those noted in other studies by Todeschini et al., where there were high significant difference between bone marrow infiltration and IPI.[14]

As regard serum LDH there was statistically high significant difference between cases with positive and negative bone marrow biopsy results, $(\mathrm{P}<0.001)$. There were statistically significant difference between positive and negative bone marrow biopsy studied cases as regard serum B2M and ESR, $(\mathrm{P}:<0.05)$.
In a study by Gupta et al., proved that, Serum B2M levels were found to be significant in patients of NHL $(p<0.05)$. Levels were significantly higher in patients with later stages (stage III and IV) than those with early stages (stage I + II), $(\mathrm{p}<0.05)$. [15]

Also in a study by Rawand and Ranan, who proved that; Serum LDH and RSR were significantly higher in NHL patients with bone marrow infiltration compared to control group $(\mathrm{P}<0.001)$.[16]

In this study 6 of the 50 specimens of NHL, both bone marrow biopsy and aspiration BMB were positive, and in 30 of 50, the two parameters were negative. Thus, the agreement level between BMB and BMA in diagnosis of NHL was $72 \%$ and discrepancies between bone marrow biopsy and aspirate were found in the remainder. In 14 of 50 specimens (28\%), BMB alone was positive.

These results were in agreement with a study by $\mathrm{Sah}$ et al., who concluded that in 13 of the 39 specimens of NHL, all the three parameters (FC, BMB, and aspirates) were positive, and in nine of 39 all the three parameters were negative. Thus, the agreement level in B-NHL was 56\% and discrepancies between immunophenotyping, $\mathrm{BMB}$, and aspirate were found in the remainder.[13]

Also this agreement with Khan et al., who work on 443 cases of different hematological disorders and found in $26 \%$ of cases bone marrow biopsy had the upper hand in diagnosis where bone marrow aspiration results were negative[17]

Also in a study by Guldeli et al., who concluded that: the examination of bone marrow biopsies increased diagnostic accuracy, contributing to prognosis especially in lymphomas and only a biopsy provided a complete assessment of bone marrow architecture and pathological pattern of distribution in case of infiltrative disorders like metastasis, lymphomas and was useful for special stains such as Immunohistochemical stains.[18]

\section{CONCOLUTION}

From all the previous results concluded that: the examination of bone marrow biopsies increased diagnostic accuracy, contributing to 
prognosis and only a biopsy provided a complete assessment of bone marrow architecture, pathological pattern of distribution in case of infiltrative disorders and was useful for special stains such as Immunohistochemical stains.

\section{Abbreviations:}

NHL, non Hodgkin's lymphoma, BMB, Bone marrow biopsy. BMA,Bone marrow aspiration. IHC, immunohistochemistry., FC: flowcytometry, DLBCL, diffuse lrge B-cell lymphoma., FL, follicular lymphoma., MZL, marginal zone lymphoma., LPL, Lymphoplasmacytic lymphoma.

\section{REFERENCES}

1- Abdel-Fattah MM and Yassine OG,:NonHodgkin's lymphomas in Alexandria, Egypt; incidence rates and trend study (1995- 2004). Eur J Cancer prev. oct; 2007: 16(5):479-85.

2- Kate R Shankland, James O Armitage, Barry W Hancock (2012): Non-Hodgkin lymphoma. Lancet 2012; 380: 848-57

3- Swerdlow SH, Campo E, Harris NL, et al. WHO classifi cation of tumours of haematopoietic and lymphoid tissues, 4th edn. Lyon,France: IARC Press, 2008.

4- Bian BJ. :Bone marrow trephine biopsy J Clin Pathol 2001; 54:737-742

5- Lewis S.M., Bain B.J. and Bates I. eds. (2006): Practical Haematology, 10th ed., pp. 1-722. Churchill Livingstone, London.

6- Naresh KN , I Lampert, R Hasserjian, D Lykidis, K Elderfield, D Horncastle, N Smith, et al, (2006 ): Optimal processing of bone marrow trephine biopsy, the Hammersmith Protocol: J. Clin. Pathol. 2006;59;903-911

7- Sovani V, Harvey C, Haynes A, McMillin A and Clark D: Bone marrow trephine biopsy involvement by lymphoma: review of histopathological features in 511 specimens and correlation with diagnostic biopsy, aspirate and peripheral blood findings. J Clin Pathol 2013; 10:2013-2015.

8- Tarek M.N. EL Bolkeney, Wael S., Heba M. and Nadia M. Mokhtar.: Evaluation of Bone Marrow in 143 Lymphomas:The Relative Frequency and
Pattern of Involvement, Secondary Myelopathies, Pitfalls and Diagnostic Validity. Journal of the Egyptian Nat. Cancer Inst.; 2008; 20(3): 17-30

9- Fatma A. Elrefaey, Gehan H. Shahin and Nesrine ElGarabawi: A morphological and Immunohistochemical study to assess bone marrow involvement in Egyptian Non Hodgkin lymphoma patients. Life Sci J.2015;13(2):1-6

10- Kumar S, Rau A, Naik R and Kini H.: Bone marrow biopsy in NHL:A morphological study. Indian Journal of pathology and microbiology; (2009)52(3): 332-338.

11- Campbell J, Seymour JF, Juneeja $S$ and Wolf M.: The Prognostic impact of bone marrow involvement in patients with DLBCL varies according to the degree of infiltration and presence of discordant marrow involvement. Eur $\mathbf{J}$ Haematol 2006; 76: 473-480.

12- Arber DA and George TI: Bone marrow biopsy involvement by non Hodgkin's lymphoma: frequency of lymphoma types, patterns, blood involvement, and discordance with other sites in 450 specimens." Am J Surg Pathol 2005; 29(12):1549-1557.

13- Sah S, E Matutes, A C Wotherspoon, R Morilla, and D Catovsky J:A comparison of flow cytometry, bone marrow biopsy, and bone marrow aspirates in the detection of lymphoid infiltration in $B$ cell disorders. Clin Pathol;2003, 56(2): 129-132.

14- Todeschini G, Secchi S, Morra E, et al. (2004): Primary mediastinal large B-cell lymphoma (PMLBCL): longterm results from a retrospective multicentre Italian experience in 138 patients treated with CHOP or MACOP-B/VACOP-B. Br J Cancer; 90:372-6.

15- Gupta G, Shalini Shaha, Nisha Marwaha, Megha Kathuriaa, Sonia Chhabraa and Rajeev Sena: Brentuximab Vedotin and Diffuse Large B-cell Lymphoma . J Hematol. 2015;4(4):242-245

16- Rawand P. Shamoon and Ranan K. Polus: Serum Lactic Dehydrogenase (LDH) Activity in Lymphomas: 
Prognostic Significance and Relationship to Presentation, Stage and Histologic Type . Zanco J. Med. Sci 2010 ;14:85-89.

17- Khan TA, Khan IA and Mahmood K. (): Diagnostic role of bone marrow aspiration and trephine biopsy in haematological practice. J Postgrad Med Inst 2014; 28(2):217-21.
18- Guldeli V., Renuka I.V., Premalatha P.,S.Indiradevi., M.Atchyutha and Krishna Divya (2015): Interpretation of Bone Marrow Trephine Biopsies in Hematological Disorders with Special Emphasis on Morphological Patterns in Lymphomas. Indian Journal of Pathology and Oncology;2(4):240-250 